Revista Científica do Instituto Agronômico do Estado de São Paulo

\title{
ESTUDO DE MATERIAIS CALCARIOS USADOS COMO CORRETIVOS DO SOLO NO ESTADO DE SÃO PAUULO. IV - O PODER RELATIVO DE NEUTRALIZAÇÃO TOTAL ${ }^{(1)}$
}

Bernardo van RaIJ $\left({ }^{2}\right)$, Seção de Fertilidade do Solo, Instituto Agronômico

\section{SINOPSE}

São apresentados dados do poder relativo de neutralização total (PRNT) de calcários utilizados como corretivos do solo no Estado de São Paulo. O PRNT é uma medida da eficiência dos calcários que conjuga o poder de neutralização total com a eficiencia relativa de partículas de diferentes tamanhos.

No trabalho é proposto o cálculo do PRNT com utilização dos teores de cálcio e magnésio e a distribuição granulométrica determinada apenas com as peneiras 10 e 50 .

Os resultados de PRNT das 25 amostras de calcários analisadas variaram de 52,3 a 93,8\% de $\mathrm{CaCO}_{3}$ equivalente, com um valor médio de $66,9 \%$.

\section{1 - INTRODUÇÃO}

Há vários anos vêm sendo aplicados a calcários no Sul do País, cálculos do "poder relativo de neutralização total" abreviado como "PRNT". O cálculo do PRNT mede a eficiência de calcários conjugando o poder de neutralização total com a eficiência relativa de partículas de diferentes tamanhos.

Nos outros estados, embora seja reconhecida a importância da granu- lometria na ação dos calcários como corretivos da acidez, cálculos da eficiência em geral limitam-se a considerar a composição química. Esta situação talvez seja decorrente da inexistência de uma maneira acessível de calcular o PRNT bem como a uma falta de divulgação desse conceito.

O cálculo do PRNT feito no Sul admite eficiência zero para partículas retidas na peneira n. ${ }^{\circ} 8$ (designação 
U.S.B.S.), eficiência de $20 \%$ para partículas retidas entre peneiras 20 e 8 , eficiência de $60 \%$ para partículas retidas entre as peneiras 60 e 20 e eficiência $100 \%$ para as partículas que passam na peneira 60 . Tal critério, utilizado no estado de Ohio, Estados Unidos (8), parece basear-se na pesquisa realizada por Pierre (6) no Estado de Alabama daquele mesmo país.

Embora a eficiência de diferentes frações granılométricas de calcários seja influenciada por diversos fatores, tais como tipo de calcário, grau de acidez do solo e tempo de reação do calcário com o solo, há uma concordância bastante grande entre diversơs autores sobre a baixa eficiência das frações mais grosseiras $(2,4,5,6)$, o que foi bem resumido na revisão feita por Barber (1) e confirmado no trabalho de Verlêngia e Gargantini (10), realizado em São Paulo.

Uma das dificuldades práticas em calcular o PRNT como é feito no Rio Granue do Sul está no conjunto de peneiras, de número 8,20 e 60 , empregadas na determinação da distribuição granulométrica, diferentes das peneiras 10 e 50 necessárias para caracterizar os calcários frente à legislação sobre corretivos em vigor no País.

Neste trabalho, o quarto de uma série sobre calcários empregados no Estado de São Paulo, é apresentado Equiv. $\mathrm{CaCO}_{3} \%$ x $[(60 \mathrm{~m} / \mathrm{p} \%$ o PRNT dos corretivos estudados nos trabalhos anteriores $(3,7,9)$. Além disso é proposta uma fórmula para cálculo de PRNT que permite utilizar os resultadơs da granulometria determinada com emprego das peneiras 10 e 50 .

\section{2 - MATERIAL E METODOS}

Foram usadas as mesmas amostras de calcários utilizadas em estudos anteriores $(3,7,9)$, com exceção de duas amostras que apresentavam somas de $\mathrm{CaO}$ e $\mathrm{MgO}$ bem inferiores ao mínimo de $38 \%$ exigido pela legislação atual sobre fertilizantes e corretivos $\left({ }^{3}\right)$. Estas amostras foram coletadas diretamente nas fontes produtoras (3).

A distribuição granulométrica dos calcários foi determinada passando cerca de $100 \mathrm{~g}$ de calcário através de peneiras de números $10,20,50 \mathrm{e}$ 60 (designação U.S.B.S.), com aberturas de malha respectivamente de 2,$00 ; 0,84 ; 0,297$ e $0,250 \mathrm{~mm}$.

Os teores de $\mathrm{CaO}$ e $\mathrm{MgO}$ foram determinados em trabalho anterior (7).

O poder relativo de neutralização total (PRNT) foi calculado pela fórmula utilizada no Rio Grande do Sul e também por uma fórmula proposta neste trabalho.

A fórmula utilizada no Rio Grande do Sul (11) é a seguinte:

PRNT $=$ $\times 1,0)+(20-60 \mathrm{~m} / \mathrm{p} \% \times 0,6)+(10-20 / \mathrm{p} \% \times 0,2)]$

100

onde: $60 \mathrm{~m} / \mathrm{p} \%$ - porcentagem de calcário que passa na peneira 60 ;

$20-60 \mathrm{~m} / \mathrm{p} \%$ - porcentagem de calcário que fica retido entre as peneiras 20 e 60 ; $10-20 \mathrm{~m} / \mathrm{p} \%$ - porcentagem de calcário que fica retido entre as peneiras 10 e 20 .

(3) Lei n. 6.138 , de 8 de novembro de 1974, regulamentada pelo Decreto n.0 75.583 em 9 de 
O equivalente carbonato de cálcalculado pela expressão:

cio, ou poder de neutralização, é

Equivalente $\mathrm{CaCo}_{3} \%=\mathrm{CaO} \% \times 1,79+\mathrm{MgO} \% \times 2,48$

A fórmula proposta neste trabalho é a seguinte:

Equiv. $\left.\left.\mathrm{CaCO}_{3} \% \times[50 \mathrm{~m} / \mathrm{p} \% \times 1,0)+10-50 \mathrm{~m} / \mathrm{p} \% \times 0,4\right)\right]$

PRNT $=\frac{}{190}$

onde: $50 \mathrm{~m} / \mathrm{p} \%$ - porcentagem de calcário que passa na peneira 50 ;

$10-50 \mathrm{~m} / \mathrm{p} \%$ - porcentagem de calcár

O equivalente carbonato de cálcio é calculado pela mesma expressão

\section{3 - RESULTADOS E DISCUSSÃO}

No quadro 1 são apresentados os resultados analíticos dos calcários que foram utilizados nos cálculos de PRNT.

Em média, os calcários apresentam características analíticas acima dos mínimơs legais para calcários, que são de $38 \%$ para soma de óxidos de cálcio e magnésio, devendo um mínimo de $50 \%$ do material passar na peneira 50 e todo ele passar na peneira 10. Merecem atenção os teores relativamente elevados de material retido na peneira 20 em diversas amostras.

No quadro 2 são apresentados os resultados calculados de poder de neutralização, da eficiência baseada na granulometria e do PRNT. Tanto a eficiência baseada na granulometria como o PRNT foram calculados pelas fórmulas dadas anteriormente.

$O$ poder de neutralização ou equivalente $\mathrm{CaCO}_{3}$ é o máximo que cada calcário contém disponível para a neutralização da acidez dơs solos. A amplitude de variação entre o maior e o menor valor apresentado atinge 24,9 .

O grau de utilização do poder de neutralização de cada calcário na neutralização da acidez dos solos irá dada anteriormente.

depender da granulometria, quantificada em termus da eficiência baseada na granulometria. Esta eficiência, pelo cálculo proposto, apresenta umá amplitude de variação de $35,5 \%$ entre o maior e o menor valor apresentado. Cabe ressaltar que a diferença para 100 da eficiência baseada na granulometria representa a porcentagem do calcáriơ que ficará inativo no solo, no prazo de poucos anos. Os dados apresentados permitem calcular um valor médio de $26,2 \%$ de calcário ineficiente.

Os dados de PRNT, também expressos em termos de $\mathrm{CaCO}_{3}$ equivalente, apresentam uma amplitude de 41,5 entre o maior e o menor valor obtido pelo cálculo proposto, o que certamente é um número que não pode ser desprezado na prática da calagem.

Com efeito, o pior calcário, com PRNT de 52,3, e o melhor, com PRNT de 93,8 devem ter ação bem diversa sobre a acidez do solo, necessitando-se $1,8 \mathrm{t}$ do primeiro para o mesmo efeito de $1 \mathrm{t}$ do segundo.

$\mathrm{Na}$ figura 1 é apresentada a excelente correlação que existe entre os valores de PRNT obtidos pelo cálculo do Rio Grande do Sul e pelo cálculo proposto. Existe uma dife- 
QUADRO 1. - Teores de cálcio e magnésio e composiçāo granulométrica dos calcários

\begin{tabular}{|c|c|c|c|c|c|c|c|}
\hline \multirow{3}{*}{$\begin{array}{c}\text { Amostra } \\
\mathrm{N}^{\circ}\end{array}$} & \multirow[b]{3}{*}{ CAO } & \multirow[b]{3}{*}{$\mathrm{MgO}$} & \multicolumn{5}{|c|}{ Composição granulométrica } \\
\hline & & & \multicolumn{4}{|c|}{ Material retido nas peneiras } & \multirow{2}{*}{$\begin{array}{c}\text { Material que passa na } \\
\text { peneira } 60\end{array}$} \\
\hline & & & 10 & 20 & 50 & 60 & \\
\hline & $\%$ & $\%$ & $\%$ & $\%$ & $\%$ & $\%$ & $\%$ \\
\hline $\begin{array}{l}1 \\
2 \\
5 \\
6 \\
7\end{array}$ & $\begin{array}{l}23,8 \\
30,1 \\
25,2 \\
23,3 \\
23,6\end{array}$ & $\begin{array}{l}15,2 \\
19,6 \\
16,5 \\
15,3 \\
15,1\end{array}$ & $\begin{array}{l}1,3 \\
0,0 \\
1,2 \\
1,4 \\
2,1\end{array}$ & $\begin{array}{l}25,9 \\
21,4 \\
27,3 \\
26,5 \\
31,3\end{array}$ & $\begin{array}{l}22,6 \\
27,6 \\
24,7 \\
22,2 \\
22,7\end{array}$ & $\begin{array}{l}8,3 \\
9,0 \\
9,0 \\
8,1 \\
7,3\end{array}$ & $\begin{array}{l}41,9 \\
42,0 \\
37,7 \\
41,8 \\
36,6\end{array}$ \\
\hline $\begin{array}{r}8 \\
9 \\
10 \\
11\end{array}$ & $\begin{array}{l}33,0 \\
26,2 \\
26,7 \\
24,4\end{array}$ & $\begin{array}{l}14,9 \\
18,4 \\
19,0 \\
17,2\end{array}$ & $\begin{array}{l}1,4 \\
1,1 \\
3,9 \\
2,7\end{array}$ & $\begin{array}{l}24,2 \\
21,0 \\
25,5 \\
24,5\end{array}$ & $\begin{array}{l}23,6 \\
20,8 \\
21,9 \\
19,6\end{array}$ & $\begin{array}{l}6,7 \\
7,9 \\
7,9 \\
7,7\end{array}$ & $\begin{array}{l}44,0 \\
49,3 \\
40,8 \\
45,5\end{array}$ \\
\hline $\begin{array}{l}12 \\
13 \\
14 \\
15 \\
16 \\
19\end{array}$ & $\begin{array}{l}26,6 \\
25,3 \\
26,0 \\
34,9 \\
34,6 \\
40,3\end{array}$ & $\begin{array}{r}14,3 \\
15,5 \\
18,1 \\
11,6 \\
12,5 \\
9,2\end{array}$ & $\begin{array}{l}4,6 \\
3,7 \\
1,0 \\
0,1 \\
0,0 \\
0,0\end{array}$ & $\begin{array}{r}32,7 \\
20,9 \\
17,0 \\
5,5 \\
7,8 \\
0,2\end{array}$ & $\begin{array}{r}21,1 \\
22,3 \\
19,7 \\
19,9 \\
26,8 \\
2,2\end{array}$ & $\begin{array}{r}6,7 \\
9,1 \\
8,5 \\
10,7 \\
11,9 \\
4,2\end{array}$ & $\begin{array}{l}34,9 \\
44,0 \\
53,7 \\
63,8 \\
\mathbf{5 3 , 5} \\
\mathbf{9 3 , 5}\end{array}$ \\
\hline $\begin{array}{l}20 \\
21 \\
22 \\
24 \\
25\end{array}$ & $\begin{array}{l}25,5 \\
26,2 \\
27,9 \\
23,5 \\
47,0\end{array}$ & $\begin{array}{r}16,9 \\
18,1 \\
18,7 \\
14,3 \\
3,7\end{array}$ & $\begin{array}{l}6,0 \\
2,1 \\
0,4 \\
5,1 \\
2,7\end{array}$ & $\begin{array}{l}25,0 \\
22,9 \\
16,0 \\
15,1 \\
21,3\end{array}$ & $\begin{array}{l}18,9 \\
20,8 \\
28,6 \\
13,7 \\
16,8\end{array}$ & $\begin{array}{r}6,8 \\
7,7 \\
10,0 \\
8,1 \\
6,7\end{array}$ & $\begin{array}{l}43,3 \\
46,6 \\
45,1 \\
57,9 \\
52,6\end{array}$ \\
\hline $\begin{array}{l}26 \\
27 \\
29 \\
31 \\
32\end{array}$ & $\begin{array}{l}45,5 \\
27,7 \\
30,0 \\
32,2 \\
42,0\end{array}$ & $\begin{array}{r}2,2 \\
16,6 \\
18,3 \\
18,2 \\
9,1\end{array}$ & $\begin{array}{l}0,0 \\
0,0 \\
0,1 \\
0,1 \\
0,6\end{array}$ & $\begin{array}{r}0,2 \\
28,5 \\
25,2 \\
7,3 \\
35,5\end{array}$ & $\begin{array}{r}7,1 \\
25,1 \\
26,1 \\
21,5 \\
19,6\end{array}$ & $\begin{array}{r}13,5 \\
7,7 \\
5,6 \\
13,2 \\
5,6\end{array}$ & $\begin{array}{l}79,1 \\
38,8 \\
43,1 \\
58,0 \\
40,7\end{array}$ \\
\hline Média & 30,1 & 14,7 & 1,7 & 20,3 & 20,6 & 8,3 & 49,1 \\
\hline
\end{tabular}

rença de $2,8 \%$ entre a média dos resultados, o que para a finalidade proposta é insignificante.

E interessante comentar sobre a validade dos fatores de eficiência propostos, de 0,4 para a fração retida entre as peneiras 10 e 50 e 1,0 para a fração que passa na peneira 50 . O comentário será feito, com auxílio dos dados apresentados por Lepsch e outros (3) e por Verlêngia e Gargantini (10) para calcários de São Paulo.

Lepsch e outros (3) apresentam dados de 19 calcários que permitem calcular as composições granulométricas médias de $18,9 \%, 10,9 \%$ e $16,7 \%$ respectivamente para materiais retidos entre peneiras $10-20$, 20-30 e 30-50.

Verlêngia e Gargantini (10) apresentam dados de incubação de dois solos, com diferentes frações granulométricas de um calcário dolomítico e um calcítico, em dois níveis de calagem. Pelo aumento dos teores de $\mathrm{Ca}^{2+}+\mathrm{Mg}^{2+}$, após dois anos de incubação, pôde-se calcular, pelos dados apresentados, eficiências mé- 
QUADRO 2. - Poder de neutralização ou equivalente $\mathrm{CaCO}_{3}$, eficiência baseada na granulometria e poder relativo de neutralizaçáo total (PRNT) dos calcários

\begin{tabular}{|c|c|c|c|c|c|}
\hline \multirow{2}{*}{$\begin{array}{c}\text { Amastra } \\
\text { N. }{ }^{\circ}\end{array}$} & \multirow{2}{*}{$\begin{array}{c}\text { Poder de } \\
\text { neutralização }\end{array}$} & \multicolumn{2}{|c|}{$\begin{array}{l}\text { Eficiência baseada na } \\
\text { granulometria }\end{array}$} & \multicolumn{2}{|c|}{ PRNT } \\
\hline & & $\begin{array}{l}\text { Pelo cálculo } \\
\text { Rio Grande } \\
\text { do Sul }\end{array}$ & $\begin{array}{l}\text { Pelo cálculo } \\
\text { proposto }\end{array}$ & $\begin{array}{l}\text { Pelo cálculo } \\
\text { Rio Grande } \\
\text { do Sul }\end{array}$ & $\begin{array}{l}\text { Pelo cálculo } \\
\text { proposto }\end{array}$ \\
\hline & $\% \mathrm{CaCO}_{3}$ & $\%$ & $\%$ & $\% \mathbf{C a C O}_{3}$ & $\% \mathrm{CaCO}_{3}$ \\
\hline $\begin{array}{l}1 \\
2 \\
5 \\
6 \\
7\end{array}$ & $\begin{array}{r}80,3 \\
102,5 \\
86,0 \\
79,7 \\
79,8\end{array}$ & $\begin{array}{l}65,6 \\
68,2 \\
63,4 \\
65,3 \\
60,9\end{array}$ & $\begin{array}{l}69,5 \\
70,7 \\
66,1 \\
69,5 \\
65,5\end{array}$ & $\begin{array}{l}52,7 \\
69,9 \\
54,5 \\
52,0 \\
52,3\end{array}$ & $\begin{array}{l}55,8 \\
72,5 \\
56,8 \\
55,4 \\
52,3\end{array}$ \\
\hline $\begin{array}{r}8 \\
9 \\
10 \\
11 \\
12\end{array}$ & $\begin{array}{l}96,1 \\
92,5 \\
94,9 \\
86,4 \\
83,1\end{array}$ & $\begin{array}{l}67,6 \\
70,7 \\
63,8 \\
66,8 \\
58,1\end{array}$ & $\begin{array}{l}69,9 \\
74,0 \\
67,7 \\
70,9 \\
63,2\end{array}$ & $\begin{array}{l}65,0 \\
65,4 \\
60,5 \\
57,7 \\
48,3\end{array}$ & $\begin{array}{l}67,2 \\
68,5 \\
64,2 \\
61,3 \\
52,5\end{array}$ \\
\hline $\begin{array}{l}13 \\
14 \\
15 \\
16 \\
19\end{array}$ & $\begin{array}{l}83,7 \\
91,5 \\
91,3 \\
93,0 \\
95,0\end{array}$ & $\begin{array}{l}67,1 \\
74,0 \\
83,3 \\
78,3 \\
97,5\end{array}$ & $\begin{array}{l}70,4 \\
76,9 \\
84,7 \\
79,3 \\
98,7\end{array}$ & $\begin{array}{l}56,2 \\
67,7 \\
76,1 \\
72,8 \\
92,6\end{array}$ & $\begin{array}{l}58,9 \\
70,4 \\
77,3 \\
73,7 \\
93,8\end{array}$ \\
\hline $\begin{array}{l}20 \\
21 \\
22 \\
24 \\
25\end{array}$ & $\begin{array}{l}87,5 \\
91,8 \\
96,4 \\
77,6 \\
93,4\end{array}$ & $\begin{array}{l}63,7 \\
68,3 \\
71,5 \\
74,0 \\
71,0\end{array}$ & $\begin{array}{l}67,9 \\
72,0 \\
73,2 \\
77,7 \\
74,0\end{array}$ & $\begin{array}{l}55,7 \\
62,7 \\
68,9 \\
57,4 \\
66,3\end{array}$ & $\begin{array}{l}59,4 \\
66,1 \\
70,6 \\
60,3 \\
69,8\end{array}$ \\
\hline $\begin{array}{l}26 \\
27 \\
29 \\
31 \\
32\end{array}$ & $\begin{array}{r}87,0 \\
90,8 \\
99,1 \\
102,7 \\
97,8\end{array}$ & $\begin{array}{l}91,5 \\
64,2 \\
66,5 \\
80,3 \\
62,5\end{array}$ & $\begin{array}{l}95,6 \\
68,2 \\
69,1 \\
82,9 \\
67,8\end{array}$ & $\begin{array}{l}79,6 \\
58,3 \\
65,9 \\
82,5 \\
61,1\end{array}$ & $\begin{array}{l}83,2 \\
61,9 \\
68,5 \\
85,1 \\
66,3\end{array}$ \\
\hline Média & 90,4 & 70,6 & 73,8 & 64,1 & 66,9 \\
\hline
\end{tabular}

dias de $16 \%, 43 \%, 67 \%$ e $97 \%$ para frações granulométricas retidas respectivamente entre as peneiras $10-20,20-30,30-50$ e $50-100$.

Combinando as informações dos dois trabalhos, pôde-se calcular como sendo de $3,0,4,7$ e $11,2 \%$ de $\mathrm{CaCl}$. a contribuição respectiva das frações granulométricas que ficam entre peneiras de 10-20, 20-30 e 30-50 ao PRNT médio dos 19 calcários do trabalho de Lepsch e outros (3), ou seja, $18,9 \%$ de $\mathrm{CaCO}_{3}$ para os $46,5 \%$ da fração dos calcários retida entre peneiras 10 e 50 , o que indica a eficiência de $40,6 \%$. Portanto, dados obtidos no Estado de São Paulo confirmam a validade do cálculo do PRNT e também da fórmula proposta neste trabalho para o seu cálculo.

O fato de apenas duas frações granulométricas bastarem para o cálculo da eficiência baseada na granulometria pode ser explicado pela 


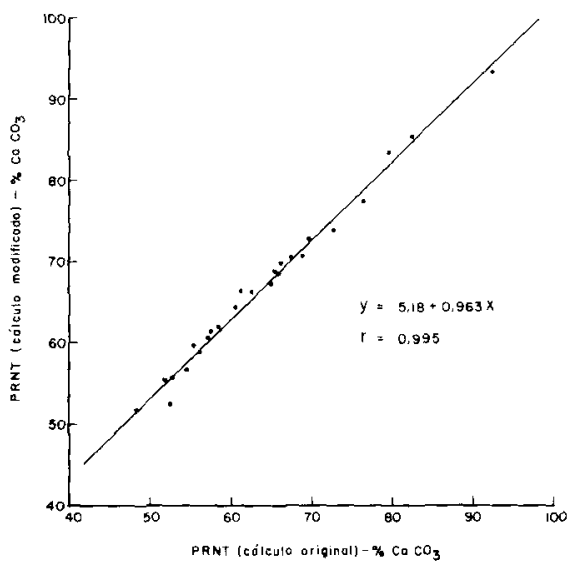

relação próxima da linear que existe entre a porcentagem cumulativa que passa em cada peneira e a abertura da peneira plotada em escala logarítmica $(1,3)$.

Duas conclusões principais podem ser tiradas do trabalho. A primeira diz respeito aos valores de PRNT dos calcários comercializados em São Paulo, que são bastante va- riáveis e de uma forma geral bastante baixos. A segunda refere-se à fórmula proposta para o cálculo do PRNT que, além de ser simples e permitir o cálculơ com os resultados da análise de calcários exigida pela legislação atual, dá resultados comparáveis aos valores obtidos por cálculo mais complexo e reflete bem a eficiência das frações granulométricas de calcários.

\section{THE RELATIVE EFFICIENCY OF LIMESTONES EMPLOYED IN NEUTRALIZING SOIL ACIDITY IN THE STATE OF SÃO PAULO}

\section{SUMMARY}

The relative efficiency of limestones was calculated with data of $\mathrm{CaO}$ and $\mathrm{MgO}$ contents and of particle size distribution determined with sieves 10 and 50 (U. S. B. S.).

The results of the 25 samples analysed varied between 52.3 and $93.8 \% \quad \mathrm{CaCO}_{3}$ equivalent, with an average of $66.9 \%$.

\section{LITERATURA CITADA}

1. BARBER, S. A. Liming materials and practices. In: PEARSON, R. W. \& ADAMS, F., ed. Soil acidity and liming. Madison, Amer. Soc. Agron., 1967. p. 125-160. (Monograph 12) 
2. BEACHER, R. L.; LONGENECKER, D. \& MERKLE, E. G. Influence of form, fineness, and amount of limestone on plant development and certain soil characteristics. Soil Sci. 73:75-82, 1952.

3. LEPSCH, I. F.; ROTTA, C. L. \& KUPPER, A. Estudo dos materiais calcários usados como corretivos do solo no Estado de São Paulo. I - Composição granulométrica. Bragantia $27: 225-238$, 1968.

4. MEYER, T. A. \& VOLK, G. W. Effect of particle size of limestones on soil reaction, exchangeable cation and plant growth. Soil Sci. 73:37-52, 1952.

5. MOTTO, H. L. \& MELSTED, M. W. The efficiency of various particle size fractions of limestone. Soil Sci. Soc. Amer. Proc. 24:488-490, 1960.

6. PIERRE, W. H. Neutralizing values and rate of reaction with acid soils of different grades and kinds of liming materials. Soil Sci. 29:137-158, 1930.

7. RAIJ, B. van; SACCHETTO, M. T. D. \& KUPPER, A. Estudo de materiais calcários usados como corretivos do solo no Estado de São Paulo. II - Composição química. Bragantia $27: 493-500,1968$.

8. TISDALE, S. L. \& NELSON, W. L. Soil fertility and fertilizers. 2nd ed. New York, Macmillan, 1966. $694 \mathrm{p}$.

9. VAIAADARES, J. M. A. S.; BATAGLIA, O. C. \& FURLANI, P. R. Estudo de materiais calcários usados como corretivos do solo no Estado de São Paulo. III - Determinação de Mo, Co, Cu, Zn, Mn e Fe. Bragantia 33:147-152, 1974.

10. VERLENGIA, F. \& GARGANTINI, H. Estudo sobre a eficiência de diferentes fraçōes granulométricas de calcário no solo. Bragantia 31:119-128, 1972.

11. VOLKWEISS, S. J. \& LUDWICK, A. E. O melhoramento do solo pela calagem. Universidade Federal do Rio Grande do Sul, Faculdade de Agronomia. 1971. 30 p. (Boletim Técnico n. ${ }^{\circ}$ ). 